\title{
GEOMETRY OF DOTS AND ROPES
}

\author{
KAREN A. CHANDLER
}

\begin{abstract}
An $\alpha$-dot is the first infinitesimal neighbourhood of a point with respect to an $(\alpha-1)$-dimensional affine space. We define a notion of uniform position for a collection of dots in projective space, which in particular holds for a collection of dots arising as a general plane section of a higher-dimensional scheme. We estimate the Hilbert function of such a collection of dots, with the result that
\end{abstract}

Theorem 1. Let $\Gamma$ be a collection of $d$ d-dots in uniform position in $\mathbb{P}^{n}$, $\alpha \geq 2$. Then the Hilbert function $h_{\Gamma}$ of $\Gamma$ satisfies

$$
h_{\Gamma}(r) \geq \min (r n+1,2 d)+(\alpha-2) \min ((r-1) n-1, d)
$$

for $r \geq 3$. Equality occurs for some $r$ with $r n+2 \leq 2 d$ if and only if $\Gamma_{\text {red }}$ is contained in a rational normal curve $C$, and the tangent directions to this curve at these points are all contained in $\Gamma$. Equality occurs for some $r$ with $(r-1) n \leq d$ if and only if $\Gamma$ is contained in the first infinitesimal neighbourhood of $C$ with respect to a subbundle, of rank $\alpha-1$ and of maximal degree, of the normal bundle of $C$ in $\mathbb{P}^{n}$.

This implies an upper bound on the degree of a subbundle of rank $\alpha-1$ of the normal bundle of an irreducible nondegenerate smooth curve of degree $d$ in $\mathbb{P}^{n}$, by a Castelnuovo argument.

\section{INTRODUCTION}

Definition 1. We define an $\alpha$-rope to be a projective scheme $X$ such that

(1) $C=X_{\mathrm{red}}$ is an irreducible nondegenerate smooth curve,

(2) the ideal sheaf $\mathscr{I}=\mathscr{I}_{X}, C$ has $\mathscr{I}^{2}=0$ and hence is an $\mathscr{O}_{C}$-module,

(3) $\mathcal{I}$ is locally free of rank $\alpha-1$ over $C$.

An $\alpha$-rope $X \subset \mathbb{P}^{n}$ corresponds naturally to a rank $\alpha-1$ subbundle $E$ of the normal bundle of its underlying curve $C$, via an exact sequence

$$
0 \rightarrow E^{*} \rightarrow \mathscr{O}_{X} \rightarrow \mathscr{O}_{C} \rightarrow 0 \text {. }
$$

For example, a 1-rope is just a curve $C \subset \mathbb{P}^{n}$, and a 2-rope is a ribbon in the sense of [BE], whereas an $n$-rope in $\mathbb{P}^{n}$ is the first infinitesimal neighbourhood of the curve in its entire normal bundle. We would like to find a lower bound for the Hilbert function $h_{X}$ of any rope $X$, and thereby obtain an upper bound for its genus. This in turn gives us an estimate for the degree of any subbundle

Received by the editors March 15, 1993.

1991 Mathematics Subject Classification. Primary 13D40, 14 H99.

Research was supported in part by a Sloan fellowship. 
$E$ of the normal bundle of a curve $C \subset \mathbb{P}^{n}$ in terms of the degree and genus of $C$ and the rank $\alpha-1$ of $E$. Applying Riemann-Roch to the exact sequence above, it follows that the degree of $E$ is $G+\alpha(1-g)-1$, where $G$ is the genus of $X$ and $g$ is the genus of $C$.

The inspiration for this problem is the following classical result due to Castelnuovo:

Theorem 2 (Castelnuovo). Let $C$ be an irreducible nondegenerate curve of degree $d$ in a projective space $\mathbb{P}^{n}$. Then the genus $g$ of $C$ satisfies

$$
g \leq(n-1)\left(\begin{array}{c}
m \\
2
\end{array}\right)+m \nu,
$$

where $m=\left[\frac{d-1}{n-1}\right]$ and $d=m(n-1)+1+\nu$.

A Castelnuovo curve is one whose genus $g$ achieves the bound of this theorem, roughly $g=\frac{d^{2}}{2(n-1)}+O(n)$. Castelnuovo obtains this upper bound on the genus by bounding from below the number of independent linear conditions imposed by the curve on hypersurfaces of each fixed degree, viz. the Hilbert function of the curve. His idea is to estimate the Hilbert function $h_{X}$ of $X \subset \mathbb{P}^{n}$ by considering a general hyperplane section $\Gamma=H \cap X$ of $X$, using the following two observations:

Lemma 3. Let $X \subset \mathbb{P}^{n}$ be a subscheme, and let $Y=X \cap H$ be a hyperplane section, where $H$ is a hyperplane not containing any component of $X$. Then

$$
h_{X}(r)-h_{X}(r-1) \geq h_{Y, H}(r) .
$$

(If $H$ is any hyperplane, then let $X^{\prime}$ be the union of the components of $X$ not lying in $H$ and $Y=X^{\prime} \cap H$ to obtain the same result.)

Proof. We may find $h_{X}(r-1)$ homogeneous polynomials of degree $r-1$ no linear combination of which vanishes on $X$. Now if we multiply each of these by the linear form $L$ that defines the hyperplane $H$, we obtain $h_{X}(r-1)$ homogeneous polynomials of degree $r$ that vanish on $H$ and such that no linear combination of them vanishes on $X$. Likewise, we may find $h_{Y, H}(r)$ homogeneous polynomials of degree $r$, no linear combination of which vanishes on either $Y$ or $H$.

Lemma 4 (Castelnuovo). Let $\mathscr{D}$ be a linear system on $C$ and let $\Gamma=H \cap C$ a hyperplane section that is general with respect to $\mathscr{D}$. Then $\Gamma$ is a collection of $d$ (reduced) points having the property that any two subsets of $\Gamma$ consisting of the same number of points imposes the same number of conditions on $\mathscr{D}$.

Such a collection of points is said to be in uniform position. It is easy to see [H1] that the uniform position of $\Gamma$ implies that its Hilbert function $h_{\Gamma}$ satisfies the following "subadditivity" property:

$$
h_{\Gamma}(r+s) \geq \min \left(h_{\Gamma}(r)+h_{\Gamma}(s)-1, d\right) .
$$

Indeed, to see this we may find subschemes $\Sigma, \Sigma^{\prime} \subset \Gamma$ of degrees $h_{\Gamma}(r), h_{\Gamma}(s)$, respectively with some point $p$ in common. Then there are forms $F, G$ of degrees $r, s$ vanishing, respectively, on $\Sigma-p, \Sigma^{\prime}-p$, with neither vanishing 
at $p$, and hence $F G$ is a form of degree $r+s$ vanishing at $\Sigma \cup \Sigma^{\prime}-p$ but not at $p$.

This "subadditivity" together with the fact that $\Gamma$ is in linear general position then implies that

$$
h_{\Gamma}(r) \geq \min (r n+1, \operatorname{deg} \Gamma)
$$

and hence we obtain a bound for $h_{X}(r)$. Thus, we get

Lemma 5 (Castelnuovo). Let $\Gamma \subset \mathbb{P}^{n}$ be a collection of $d$ points in uniform position. Then $h_{\Gamma}(r) \geq \min (r n+1, d)$. Moreover, we have equality for some $r$ with $r n+2 \leq d$ if and only if $\Gamma$ lies on a rational normal curve.

In [EH1], Eisenbud and Harris extend Castelnuovo's result to ribbons (2ropes). They show that if $\Gamma \subset \mathbb{P}^{n}$ is a general hyperplane section of a ribbon in $\mathbb{P}^{n+1}$, then $\Gamma$ is in linearly general position in the sense that the linear span of any subscheme $\Sigma$ of $\Gamma$ has dimension at least $\min (\operatorname{deg} \Sigma-1, n)$. Indeed, more generally such a $\Gamma$ is in uniform position in the sense that if two subschemes $\Sigma_{1}, \Sigma_{2}$ of $\Gamma$ have the same degree and the same number of reduced points, then for all $r, \Sigma_{1}$ and $\Sigma_{2}$ impose the same number of conditions on $r$-ics. Eisenbud and Harris then prove that any collection $\Gamma$ of $d$ arrows (2-dots) in linearly general position in $\mathbb{P}^{n}$ has Hilbert function at least

$$
h_{\Gamma}(r) \geq \min (r n+1,2 d),
$$

and that equality occurs for some $r$ with $r n+1 \leq 2 d$ if and only if $\Gamma$ is contained in a rational normal curve.

In this paper, we generalise the Castelnuovo argument to $\alpha$-ropes $X$. We start in section 2 by examining uniform position properties of a general hyperplane section $\Gamma$ of $X$. Necessarily, it is no longer the case that all subschemes of $\Gamma$ of the same degree impose the same number of conditions on a linear system $\mathscr{D}$. Indeed, we'll see that the minimal Hilbert function of a collection of $\alpha$-dots does depend on $\alpha$ and hence that subschemes of $\Gamma$ containing "fatter" dots will impose more conditions on $\mathscr{D}$ than, for example, just reduced points. Nonetheless, general subschemes of sufficiently similar shape will impose the same number of conditions.

Next in section 3, we examine the Hilbert functions of collections of points in uniform position. From the uniform position property, we get a formula for $h_{\Gamma}(r+1)$ in terms of $h_{\Gamma}(r)$ as before; again if we join at a point two subschemes, one of which is independent in degree $r$ and the other in degree $s$, they form a subscheme that is independent in degree $r+s$. But, further, we find that if two such subschemes are joined at several points then these points may be "promoted" to dots of higher degree. Hence, it follows that $h_{\Gamma}(r)$ grows as at least $(\alpha-1) n$, for small $r$.

In section 4, we consider Hilbert functions of ropes. We start by looking at ropes over a rational normal curve and find the $\alpha$-rope of minimal Hilbert function. Then, using the results of section 3 , we find a lower bound on the Hilbert function of an $\alpha$-rope over a curve of degree $d$ in $\mathbb{P}^{n}$. An $\alpha$-rope $X$ can approach this bound if and only if the reduced curve $C$ is a Castelnuovo curve and a general hyperplane section of $X$ lies on the $\alpha$-rope of minimal Hilbert function over a rational normal curve. 
Acknowledgments. This problem was suggested to me by Joe Harris. The ideas in this paper arose from discussions with Joe Harris and David Eisenbud.

\section{UNIFORM POSITION}

Definition 2. $A k$-dot is a scheme isomorphic to the first infinitesimal neighbourhood of a point in $\mathbb{A}_{\mathbb{C}}^{k-1}$, i.e., $\operatorname{Spec}\left(\mathbb{C}\left[z_{1}, \ldots, z_{k-1}\right] /\left(z_{1}, \ldots, z_{k-1}\right)^{2}\right)$. Thus such a scheme is given by a reduced point $p$ and its $(k-1)$-dimensional Zariski tangent space $V$; we denote this $k$-dot by $p(V)$. Where appropriate we denote by $p(k)$ any $k$-dot at the point $p$. If $k \leq 0$ we define a $k$-dot $p(k)=\emptyset$.

So, a $k$-dot has degree $k$, e.g., a 1-dot is a (reduced) point, and a 2-dot or arrow is a point together with a tangent line.

Definition 3. We say that a zero-dimensional projective scheme imposes independent conditions on a linear system $\mathscr{D}$ (or briefly, that the subscheme is $\mathscr{D}$-independent) if it imposes as many conditions on $\mathscr{D}$ as its degree.

Let $X$ be an $\alpha$-rope in $\mathbb{P}^{n}$ with $C=X_{\text {red }}$ an irreducible nondegenerate curve. We now consider the question: how "uniform" is the position of a general hyperplane section $\Gamma$ of $X$ ? More specifically, given that some subscheme of $\Gamma$ imposes independent conditions on some linear system $\mathscr{D}$, we would like to know what other subschemes will be independent as well.

Lemma 6 (Uniform Position). Let $\mathscr{D}$ be a linear system on $X$ and $\Gamma=H \cap X$ a hyperplane section general with respect to $\mathscr{D}$. Suppose that a subscheme

$$
\left\{p_{1}\left(k_{1}\right), \ldots, p_{m}\left(k_{m}\right)\right\} \subset \Gamma
$$

of $\Gamma$ imposes at least $\nu$ independent conditions on $\mathscr{D}$. Then for any reduced points $q_{1}, \ldots, q_{m}$ of $\Gamma$ there is a subscheme

$$
\left\{q_{1}\left(k_{1}\right), \ldots, q_{m}\left(k_{m}\right)\right\} \subset \Gamma
$$

of $\Gamma$ imposing at least $\nu$ independent conditions on $\mathscr{D}$.

Proof. Let

$$
U=\{\text { hyperplanes transverse to } C\} \subset \mathbb{P}^{n *},
$$

and let $I \subset C^{m} \times U$ be the subvariety

$$
I=\left\{\left(p_{1}, \ldots, p_{m} ; H\right): H \in U, p_{i} \in H \cap C\right\} .
$$

By the uniform position property for hyperplane sections of curves [ACGH], $I$ is irreducible of dimension $n$. Now if $J \subset I$ is defined by

$J=\left\{\begin{array}{l|l}\left(p_{1}, \ldots, p_{m} ; H\right) & \begin{array}{l}\text { for every } V_{1}, \ldots, V_{m} \text { with } \operatorname{dim} V_{i}=k_{i} \text { and } \\ V_{i} \subset T_{p}(H \cap X) \text { we have }\left\{p_{1}\left(V_{1}\right), \ldots, p_{m}\left(V_{m}\right)\right\} \\ \text { imposes }<\nu \text { conditions on } \mathscr{D}\end{array}\end{array}\right\}$,

then either $J=I$ or $\operatorname{dim} J<\operatorname{dim} I$, in which case $J$ projects onto a proper subvariety of $U$.

Definition 4. We will say that a collection $\Gamma$ of dots satisfying the conclusions of the previous two lemmas is in uniform position with respect to the linear system $\mathscr{D}$. 
Hence we may restate the lemma as

Lemma $6^{\prime}$. Let $\mathscr{D}$ be a linear system on $X$ and $\Gamma=H \cap X$ a hyperplane section general with respect to $\mathscr{D}$. Then $\Gamma$ is in uniform position with respect to $\mathscr{D}$.

Lemma 7. Let $\Gamma$ be a collection of dots and $\mathscr{D}$ a linear system. Let $\{p(V)$, $q(W)\} \cup \Sigma$ be a subscheme of $\Gamma$ that is $\mathscr{D}$-independent. Suppose that $W^{\prime} \supset W, \operatorname{codim}\left(W, W^{\prime}\right)=1$ such that $q\left(W^{\prime}\right) \subset \Gamma$ and $\left\{p, q\left(W^{\prime}\right)\right\} \cup \Sigma$ is $\mathscr{D}$ independent. Then there exists $V^{\prime} \subset V$ with $\operatorname{codim}\left(V^{\prime}, V\right)=1$ so that

$$
\left\{p\left(V^{\prime}\right), q\left(W^{\prime}\right)\right\} \cup \Sigma
$$

is also $\mathscr{D}$-independent.

Proof. Note that a subscheme of the form $p(k) \cup \Sigma$ of $\Gamma$ is $\mathscr{D}$-independent if and only if $p \cup \Sigma$ is $\mathscr{D}$-independent and $p(\alpha) \cup \Sigma$ imposes at least $k$ more conditions on $\mathscr{D}$ than does $\Sigma$.

Let $k=\operatorname{dim} V+1$ and $l=\operatorname{dim} W+1$. Since $\{p(V), q(W)\} \cup \Sigma$ is $\mathscr{D}$-independent, we have that this subscheme imposes $k+l$ more conditions on $\mathscr{D}$ than $\Sigma$ alone. Then of course $\left\{p(V), q\left(W^{\prime}\right)\right\} \cup \Sigma$ imposes at least $k+l$ more conditions on $\mathscr{D}$ than $\Sigma$ does. Now if $\left\{p, q\left(W^{\prime}\right)\right\} \cup \Sigma$ is $\mathscr{D}$ independent then $\left\{p(V), q\left(W^{\prime}\right)\right\} \cup \Sigma$ imposes at least $k-1$ more conditions on $\mathscr{D}$ than $\left\{q\left(W^{\prime}\right)\right\} \cup \Sigma$. Hence, there exists $V^{\prime} \subset V, \operatorname{codim}\left(V^{\prime}, V\right)=1$ so that $\left\{p\left(V^{\prime}\right), q\left(W^{\prime}\right)\right\} \cup \Sigma$ is $\mathscr{D}$-independent.

Corollary 8. Let $\Gamma$ be in uniform position with respect to the linear system $\mathscr{D}$. Suppose there is a subscheme

$$
\left\{q(l), p(k), p_{1}\left(k_{1}\right), \ldots, p_{m}\left(k_{m}\right)\right\}, \quad k_{i} \geq 1, l \geq 0, k \geq 1,
$$

of $\Gamma$ which imposes independent conditions on $D$ but that every subscheme of the form

$$
\left\{q(l+1), p(k-1), p_{1}\left(k_{1}\right), \ldots, p_{m}\left(k_{m}\right)\right\}
$$

fails to impose independent conditions on $\mathscr{D}$. Let

$$
k_{i}^{\prime}= \begin{cases}k_{i}, & \text { if } k_{i} \geq k \\ 1, & \text { otherwise } .\end{cases}
$$

Then every

$$
\left\{q(l+1), p(1), p_{1}\left(k_{1}^{\prime}\right), \ldots, p_{m}\left(k_{m}^{\prime}\right)\right\}
$$

fails to impose independent conditions on $\mathscr{D}$.

Proof. Let us call $\Sigma=\left\{p_{1}\left(k_{1}\right), \ldots, p_{m}\left(k_{m}\right)\right\}$.

By Lemma 7 , given that

$$
\{p(k-1), q(l+1)\} \cup \Sigma
$$

fails to be $\mathscr{D}$-independent (for all $(k-1)$-dots $p(k-1) \subset \Gamma$ at $p$, and all $(l+1)$-dots $q(l+1) \subset \Gamma$ at $q)$, we have that

$$
\{p, q(l+1)\} \cup \Sigma
$$


fails to be $\mathscr{D}$-independent. Let $p_{i}\left(k_{i}\right) \in \Sigma$ with $k_{i} \leq k-1$. For convenience suppose $i=1$. By uniform position we have that

$$
\left\{q(l), p, p_{1}(k), p_{2}\left(k_{2}\right), \ldots, p_{m}\left(k_{m}\right)\right\}
$$

is $\mathscr{D}$-independent (switching $p$ and $p_{1}$ ), and yet

$$
\left\{q(l+1), p, p_{1}(k-1), p_{2}\left(k_{2}\right), \ldots, p_{m}\left(k_{m}\right)\right\}
$$

is not $\mathscr{D}$-independent (since $k_{1} \leq k-1$ ), so by the preceding argument

$$
\left\{q(l+1), p(k-1), p_{1}, p_{2}\left(k_{2}\right), \ldots, p_{m}\left(k_{m}\right)\right\}
$$

fails to be $\mathscr{D}$-independent. Now, applying this argument to each $k_{i} \leq k-1$ the result follows.

Promotion. Now let $\Gamma \subset \mathbb{P}^{n}$ be a collection of $\alpha$-dots in uniform position. We now develop tools to estimate the growth of the Hilbert function of $\Gamma$.

Lemma 9 ("Promotion"). Let $\Sigma_{1}, \Sigma_{2}$ be subschemes of $\Gamma$, and let $t \leq n$. Suppose that the subscheme

$$
\left\{p_{1}\left(k_{1}\right), \ldots, p_{t}\left(k_{t}\right)\right\}
$$

imposes independent conditions on hypersurfaces of degree $r+s$. Suppose that

$$
\left\{q(k), p_{1}, \ldots, p_{t}\right\} \cup \Sigma_{1} \subset \Gamma
$$

imposes $k$ more conditions on hypersurfaces of degree $r$ than

$$
\left\{p_{1}, \ldots, p_{t}\right\} \cup \Sigma_{1} .
$$

Suppose also that

$$
\left\{q, p_{1}, \ldots, p_{t}\right\} \cup \Sigma_{2}
$$

imposes one more condition on hypersurfaces of degree $s$ than

$$
\left\{p_{1}, \ldots, p_{t}\right\} \cup \Sigma_{2} .
$$

Let $k_{i}^{\prime}=\max \left(k_{i}, k\right)$. Then

$$
\left\{q(k), p_{1}\left(k_{1}^{\prime}\right), \ldots, p_{t}\left(k_{t}^{\prime}\right)\right\} \cup \Sigma_{1}^{\prime} \cup \Sigma_{2}^{\prime}
$$

imposes independent conditions on hypersurfaces of degree $r+s$, for general subschemes $\Sigma_{1}^{\prime}, \Sigma_{2}^{\prime} \subset \Gamma$ of the same shape, respectively, as $\Sigma_{1}, \Sigma_{2}$.

Proof. We can find $F_{1}, F_{2}, \ldots, F_{k}$ of degree $r$, each vanishing on the subscheme $\left\{p_{1}, p_{2}, \ldots, p_{t}\right\} \cup \Sigma_{1}$ and so that no linear combination of them vanishes on $q(\alpha)$. We can find $G$ of degree $s$ vanishing on $\left\{p_{1}, p_{2}, \ldots, p_{t}\right\} \cup \Sigma_{2}$ and not at $q$. Thus $F_{j} G$ vanishes on $\left\{p_{1}(\alpha), p_{2}(\alpha), \ldots, p_{t}(\alpha)\right\} \cup \Sigma_{1} \cup \Sigma_{2}$ for each $i$, and no linear combination of the $F_{j} G$ vanishes at $q(\alpha)$. Now by uniform position the same holds if we exchange $q$ for any $p_{i}, i \leq t$, in case $k_{i} \leq k$. 
Corollary 10. Suppose that

$$
\Sigma \cup\left\{p_{1}, \ldots, p_{n}\right\} \subset \Gamma
$$

imposes independent conditions in degree $r$. If $\Sigma$ contains at least one $k$-dot, then if $\Sigma^{\prime} \subset \Gamma$ is a general subscheme of the same shape as $\Sigma$ then

$$
\Sigma^{\prime} \cup\left\{p_{1}(k), \ldots, p_{n}(k)\right\}
$$

imposes independent conditions in degree $r+1$.

Our strategy for estimating the Hilbert function of a collection $\Gamma$ of $\alpha$-dots in uniform position will be to find, for each $r$, a subscheme $\Sigma$ of $\Gamma$ that is $r$ independent and is maximal with respect to this property; i.e., $h_{\Gamma}(r)=h_{\Sigma}(r)=$ $\operatorname{deg} \Sigma$. If such a $\Sigma$ contains an $\alpha$-dot and at least $n$ reduced points then the promotion lemma implies that

$$
h_{\Gamma}(r+1) \geq h_{\Gamma}(r)+(\alpha-1) n .
$$

\section{ESTIMATE OF THE HILBERT FUNCTION OF DOTS IN UNIFORM POSITION}

Throughout this section, $\Gamma$ will be a collection of $d \geq n+1 \alpha$-dots in uniform position in $\mathbb{P}^{n}$, in the sense of section 2 . We will now establish that the Hilbert function $h_{\Gamma}$ of $\Gamma$ satisfies the lower bound:

$$
h_{\Gamma}(r) \geq \begin{cases}n+1, & \text { if } r=1, \\
2 n+1+(\alpha-2)(n-1)-\left(\begin{array}{c}
\alpha-2 \\
2
\end{array}\right), & \text { if } r=2, \\
\min (r n+1,2 d)+(\alpha-2) \min ((r-1) n-1, d), & \text { if } r \geq 3\end{cases}
$$

First, we verify this for $r=2,3$ using a combination of the "promotion" lemma with special tricks. Then we use induction to deduce the bound in higher degrees.

Computations in degrees 2 and 3. Let $\Gamma \subset \mathbb{P}^{n}$ be a collection of $\alpha$-dots in uniform position.

Let $p_{1}, \ldots, p_{n+1} \in \Gamma$. According to the linear general position of $\Gamma$ the subscheme

$$
\left.\left\{p_{n+1}(\alpha), p_{n}, \ldots, p_{\alpha}\right)\right\} \subset \Gamma
$$

(which we will take to mean just $\left\{p_{n+1}(\alpha)\right\}$ in case $\alpha=n+1$ ) is linearly independent, so by "promotion",

$$
\left\{p_{n+1}(\alpha), p_{n}(\alpha), \ldots, p_{\alpha}(\alpha)\right\} \subset \Gamma
$$

(respectively, $\left.\left\{p_{n+1}(\alpha)\right\}\right)$ is quadratically independent. Also, a

$$
\left\{p_{\alpha-1}(\alpha-1), p_{\alpha}, \ldots, p_{n+1}\right\} \subset \Gamma
$$

is linearly independent so again by the lemma

$$
\left\{p_{\alpha-1}(\alpha-1), p_{\alpha}(\alpha), \ldots, p_{n+1}(\alpha)\right\} \subset \Gamma
$$

is also quadratically independent. Indeed, for each $i \leq \alpha-1$ a

$$
\left\{p_{i}(i), p_{i+1}, \ldots, p_{n+1}\right\} \subset \Gamma
$$


is linearly independent so inductively applying the lemma we wind up with a

$$
\left\{p_{n+1}(\alpha), \ldots, p_{\alpha}(\alpha), p_{\alpha-1}(\alpha-1), \ldots, p_{2}(2), p_{1}(1)\right\} \subset \Gamma
$$

that is 2-independent. Hence,

$$
h_{\Gamma}(2) \geq \alpha(n+1)-\left(\begin{array}{l}
\alpha \\
2
\end{array}\right) .
$$

Immediately, by the promotion lemma, we get that $\left\{p_{n+1}(\alpha), \ldots, p_{1}(\alpha)\right\}$ imposes independent conditions on cubics, and hence $h_{\Gamma}(3) \geq \alpha(n+1)$. (Indeed, it is easy to show that a subscheme of $\Gamma$ of the form

$$
\left\{p_{n-1}\left(k_{n}\right), \ldots, p_{1}\left(k_{1}\right), q_{0}(\alpha), q_{1}(\alpha), \ldots, q_{n}(\alpha)\right\}
$$

is 3-independent if $k_{i}=\min (i, \alpha-1)$, but we will derive a stronger result.)

Let us now make some further observations about quadrics.

Lemma $11\left(\mathbb{P}^{3}\right)$. Given $p, p_{1}, \ldots, p_{5} \in \mathbb{P}^{3}$, in linear general position there is a unique quadric $Q$ that is singular at $p$ and vanishes at $p_{1}, \ldots, p_{5}$.

Remark. As we will see in section 4 , if $C$ is the twisted cubic determined by these 6 points, then $Q$ is the quadric that vanishes on the "canonical" ribbon determined by $C$ and $p$.

Proof. $\left\{p(4), p_{1}, \ldots, p_{5}\right\}$ impose 9 conditions on quadrics in $\mathbb{P}^{3}$.

Corollary 12. Given $n+3$ points $p_{1}, \ldots, p_{n+3} \in \mathbb{P}^{n}$ in linear general position, there is a unique quadric $Q$ that is singular at $p_{1}, \ldots, p_{n-2}$ and vanishes at $p_{n-1}, \ldots, p_{n+3}$.

Remark. Likewise, $Q$ is the quadric that vanishes on the "canonical" rope determined by the rational normal curve $C$ through the $n+3$ points, and with basepoints the given $n-2$ points.

Proof. If $\left(X_{0}, X_{1}, X_{2}, X_{3}\right)$ is the ideal of the $n-2$ points then the ideal of the $n-2$ dots is exactly $\left(X_{0}, X_{1}, X_{2}, X_{3}\right)^{2}$. By the lemma, exactly one of these vanishes on an additional 5 points in linear general position.

To start on the Hilbert function in degree three, let us first consider the case $\alpha=n+1$. We will find a subscheme of $\Gamma$ having $2 n-1$ reduced points and imposing at least $2 n^{2}+2$ conditions on cubics. To warm up, let us first find a subscheme having $2 n$ reduced points and imposing $2 n^{2}+2$ conditions on cubics. Take $2 n(n+1)$-dots, say $\Gamma_{0}$, of $\Gamma$. Now, fix $n+1$ of these, say $q_{1}, \ldots, q_{n+1}$. For a cubic or a quadric to be singular at all of these points it must contain every line between any pair of them, by Bezout's theorem, since it meets each such line to degree at least 4 . Moreover, this is an equivalent condition by linear general position. So, let $Y$ be the union of these lines. Let $\Sigma=p_{1}, \ldots, p_{n-1}$ be the remaining points, and let $K$ be the $(n-2)$-plane spanned by the $q_{i}$ 's, and let $Z \subset K$ be the $(n-1)$-dots at the $q_{i}$ 's. Let $H$ be a general hyperplane containing $K$.

Claim 13. We have that

(1) $Z$ imposes independent conditions on cubics in $K=\mathbb{P}^{n-2}$.

(2) No quadric in $H=\mathbb{P}^{n-1}$ contains $Y \cap H$.

(3) No quadric in $\mathbb{P}^{n}$ contains $Y$. 
The claim implies that

$$
\begin{aligned}
h_{\Gamma_{0}, \mathbb{P}^{n}}(3) & \geq h_{Z \cup(Y \cap H), H}(3)+h_{Y, \mathbb{P} n}(2) \\
& \geq h_{Z, K}(3)+h_{Y \cap H, H}(2)+h_{Y, \mathbb{P} n}(2) \\
& =(n-1)^{2}+\left(\begin{array}{c}
n+1 \\
2
\end{array}\right)+\left(\begin{array}{c}
n+2 \\
2
\end{array}\right) \\
& =2 n^{2}+2
\end{aligned}
$$

and hence we need now only verify the conditions of the claim to get our bound on $h_{\Gamma_{0}}$.

We have already seen that the first and third conditions hold, so let us prove the middle one. We can write $Y=\bigcup l_{i j}$ where $l_{i j}$ is the line between $\dot{q}_{i}$ and $q_{j}$. Then $Y \cap H=\left\{r_{i j}\right\}$ where $r_{i j}=l_{i j} \cap H$. Our goal is to prove that the points $r_{i j}$ impose independent conditions on quadrics, and hence we seek for each $i, j$ a quadric that vanishes on each $r_{k, l}$ other than $r_{i j}$ and fails to vanish at $r_{i j}$. For each $i, 1 \leq i \leq n+1$, let $H_{i}$ be the hyperplane of $\mathbb{P}^{n}$ spanned by $\left\{p_{j}: j \neq i\right\}$. Then $H_{i}$ does not contain $r_{i j}$ by linear general position. That is, if $H_{i}$ did contain $r_{i, j}$, then it would contain the whole line between $r_{i, j}$ and $q_{j}\left(r_{i, j} \neq q_{j}\right.$ since $H$ does not contain any $\left.q_{j}\right)$. But this is ruled out by the fact that $H_{i}$ does not contain $q_{i}$, which is on that line. Hence the union of the two hyperplanes of $H$ given by $H \cap H_{1}$ and $H \cap H_{2}$ gives a (reducible) quadric in $H$ as desired.

Let us now prove that actually $2 n-1(n+1)$-dots impose $2 n^{2}+2$ conditions on cubics. To see this, let $H$ be the hyperplane spanned by some $p_{1}, \ldots, p_{n} \in \Gamma$. Then

Claim 14. $\Gamma_{0}=\left\{q_{1}(n+1), \ldots, q_{n-1}(n+1), p_{1}(H), \ldots, p_{n}(H)\right\}$ imposes independent conditions on cubics.

Proof. Set $q_{n}=p_{n}$ and $q_{n+1}=p_{n-1}$ in the above, so that at least

$$
\left\{q_{1}(n+1), \ldots, q_{n-1}(n+1), p_{1}(K), \ldots, p_{n-2}(K), p_{n-1}(H), p_{n}(H)\right\}
$$

imposes independent conditions on cubics, where $K$ is the $(n-2)$-plane spanned by $p_{1}, \ldots, p_{n-1}$. In particular the subscheme

$$
\left\{r_{i, j}\right\}_{i, j \leq n-1} \cup\left\{p_{1}(K), \ldots, p_{n-2}(K), p_{n-1}(H), p_{n}(H)\right\}
$$

imposes independent conditions on cubics in $H$, with $r_{i, j}$ 's as before, $i, j<n$. For each $k$ with $1 \leq k \leq n-2$ we should find a cubic in $H$ that vanishes on $\left\{r_{i, j}\right\}_{i, j \leq n-1}$, is singular at $p_{i}$ 's for $i \neq k$, vanishes on $p_{k}(K)$, but fails to be singular at $p_{k}$. Let $H_{1}$ be the span of $q_{1}, \ldots, q_{n-1}, p_{n}$, so $H_{1} \cap H$ contains the $r_{i, j}$ 's for $i, j \leq n-1$. Let $H_{2}=K$, so $H_{2}$ contains $p_{i}(K)$ for each $i<n$. Let $H_{3}$ contain $p_{i}, i \neq k$. Then the cubic on $H$ given by $H_{i} \cap H$ is singular on $p_{i}$ 's for $i \neq k$, vanishes on $q_{k}(K)$ but fails to be singular at $q_{k}$, vanishes on $r_{i, j}$ for $i, j \leq n-1$.

Lemma 15. A collection of $2 n-1 \quad(n+1)$-dots in linear general position in $\mathbb{P}^{n}$ imposes at least $2 n^{2}+2$ conditions on cubics. 
Proof. We have

$$
\left\{p_{1}(n+1), \ldots, p_{n-1}(n+1), p_{n}(H), \ldots, p_{2 n-1}(H)\right\}
$$

is 3-independent, where $H$ is the hyperplane spanned by $\left\{p_{n}, \ldots, p_{2 n-1}\right\}$. Now we may find a quadric $Q$ that vanishes on $\left\{p_{1}(n+1), \ldots, p_{n-1}(n+1)\right.$, $\left.p_{n}, p_{n+1}\right\}$ but fails to vanish on $p_{n+2}$.

(Indeed, we may take $Q$ given by the two hyperplanes $\operatorname{span}\left(p_{1}, \ldots, p_{n}\right)$ and $\operatorname{span}\left(p_{1}, \ldots, p_{n-1}, p_{n+1}\right)$.)

Then if $L$ is a linear form vanishing on $p_{n}, \ldots, p_{2 n-1}$, we have that $Q L$ vanishes on

$$
\left\{p_{1}(n+1), \ldots, p_{n+1}(n+1), p_{n+2}(H), \ldots, p_{2 n-1}(H)\right\}
$$

and yet fails to vanish on $p_{n+2}(n+1)$. Similarly for $j=n, n+1$ we may find cubics vanishing on $p_{i}(n+1), 1 \leq i \leq n+2, i \neq j$, and on $p_{i}(H), n \leq i \leq$ $2 n-1$, and failing to vanish on $p_{j}(n+1)$. Hence

$$
\left\{p_{1}(n+1), \ldots, p_{n+2}(n+1), p_{n+3}(H), \ldots, p_{2 n-1}(H)\right\}
$$

is 3-independent.

Corollary 16. A collection of $2 n-1 \quad \alpha$-dots in linear general position in $\mathbb{P}^{n}$ imposes at least $3 n+1+(\alpha-2)(2 n-1)$ conditions on cubics.

We would like to show now that these bounds can be attained only by a collection of points lying on a rational normal curve. But first, some lemmas.

Lemma 17. Suppose that $\Gamma$ is a collection of arrows and points such that $\Gamma_{\text {red }}$ is a collection of points in linear general position, and $\operatorname{deg} \Gamma \geq 3 n+2$. If $\Gamma$ imposes only $3 n+1$ conditions on cubics then it is contained in a rational normal curve.

Proof: By the result of Eisenbud-Harris [EH1] it suffices to prove that no subscheme of $\Gamma$ of degree $n+1$ lies in a hyperplane. Let $\Sigma \subset \Gamma$ be a subscheme of minimal degree with respect to the property of not imposing independent conditions on cubics. So $\Sigma$ contains a total of $d$ points with arrows at some $m$ of them, where $m+d=\operatorname{deg} \Sigma \leq 3 n+2$. Without loss of generality, $m \geq n+2$ and $d>n+2$ or else we are already done, by Lemma 15 . Suppose that there is a hyperplane $H$ that contains a subscheme of $\Sigma$ of degree $n+1$, say

$$
\left\{p_{1}(2), \ldots, p_{t}(2), p_{t+1}, \ldots, p_{n+1-t}\right\}
$$

(where $t \geq 1$ by the linear general position of the points). If $\Sigma$ has only $t$ arrows, then we may put another $2 n$ points of $\Sigma$ on two hyperplanes $H_{1}, H_{2}$ so that $H \cup H_{1} \cup H_{2}$ contains a subscheme of $\Sigma$ of degree $\operatorname{deg} \Sigma-1$, hence contains an additional point of $\Gamma$, contrary to linear general position of the points.

Hence we may assume that there is an additional arrow, say $p_{d}(2) \subset \Sigma$. Find hyperplanes $H_{1}, H_{2}$ each containing exactly $n$ reduced points of $\Sigma$ and such that $H_{1} \cup H_{2} \cup H$ contains a subscheme of $\Sigma$ of degree $\operatorname{deg} \Sigma-1$. Choose $H_{2}$ to contain $p_{d-n-1}, \ldots, \hat{p}_{i}, \ldots, p_{d}$ and $p_{d} \notin H_{1}$. Since $H_{1} \cup H_{2} \cup H$ contains a 3-independent subscheme of $\Sigma$ of degree one less than that of $\Sigma$ this implies that $\Sigma \subset H_{1} \cup H_{2} \cup H$. In particular $p_{d}(2) \subset H_{2}$. But varying $i$ with $d-n-1 \leq i \leq d-1$, and likewise varying $H_{2}$ we get that $p_{d}(2)$ is contained in 
every hyperplane containing $p_{d}$ and $n-1$ of the points $p_{d-n-1}, \ldots, p_{d-1}$. By linear general position, however, the intersection of such hyperplanes is exactly the point $p_{d}$, contradiction.

Corollary 18. If $\left\{p_{1}, \ldots, p_{d}\right\}$ is a collection of points in linear general position in $\mathbb{P}^{n}$ and $m+d \leq 3 n+1$ for some $m$ then

$$
\left\{p_{1}(n+1), \ldots, p_{m}(n+1), p_{m}, \ldots, p_{d}\right\}
$$

is 3-independent. Moreover, if $p_{1}$ is not in the rational normal curve determined by $p_{2}, \ldots, p_{n+4}$ then

$$
\left\{p_{1}(n+1), \ldots, p_{m+1}(n+1), p_{m+2}, \ldots, p_{d}\right\}
$$

is 3-independent.

Proof. Suppose that

$$
\left\{p_{1}(n+1), \ldots, p_{m}(n+1), p_{m+1}, \ldots, p_{d}\right\}
$$

fails to be 3-independent, for some minimal $m$. We have that

$$
\left\{p_{1}(n+1), \ldots, p_{m-1}(n+1), p_{m}, \ldots, p_{d}\right\}
$$

is 3-independent, hence there is some arrow $p_{m}\left(l_{m}\right) \subset p_{m}(n+1)$ so that

$$
\left\{p_{1}(n+1), \ldots, p_{m-1}(n+1), p_{m}\left(l_{m}\right), \ldots, p_{d}\right\}
$$

is 3-dependent. However,

$$
\left\{p_{1}(n+1), \ldots, p_{m-2}(n+1), p_{m-1}, p_{m}\left(l_{m}\right) \ldots, p_{d}\right\}
$$

is 3-independent, so there is some arrow $p_{m-1}\left(l_{m-1}\right) \subset p_{m-1}(n+1)$ for which

$$
\left\{p_{1}(n+1), \ldots, p_{m-2}(n+1), p_{m-1}\left(l_{m-1}\right), p_{m}\left(l_{m}\right) \ldots, p_{d}\right\}
$$

is 3-dependent. Continuing this line of argument, we get that there is a collection

$$
\left\{p_{1}\left(l_{1}\right), \ldots, p_{m}\left(l_{m}\right), \ldots, p_{d}\right\}
$$

of arrows and points that imposes only $d+m-1$ conditions on cubics. Thus either $d+m=3 n+2$ and the points and arrows are on a rational normal curve, or else $d+m \geq 3 n+3$.

Corollary 19. A collection $\Gamma$ of $d$ a-dots in uniform position in $\mathbb{P}^{n}$ imposes at least $\min (3 n+1,2 d)+(\alpha-2) \min (2 n-1, d)$ conditions on cubics. If $2 d>3 n+1$, we have equality only if the reduced points of $\Gamma$ lie on a rational normal curve, and at each reduced point $p$ of $\Gamma$ the tangent direction to that curve at $p$ is contained in the Zariski tangent space to $\Gamma$ at $p$.

Proof. It suffices to prove this for $d \leq 2 n-1$. Let $\Gamma=\left\{p_{1}(\alpha), \ldots, p_{d}(\alpha)\right\}$ be in uniform position, where $d \leq 2 n-1$. We may find $\Gamma^{\prime}$, such that

$$
\Gamma \supset \Gamma^{\prime}=\left\{p_{1}(\alpha), \ldots, p_{m}(\alpha), p_{m+1}(\alpha-1), \ldots, p_{d}(\alpha-1)\right\}
$$

imposes independent conditions on cubics, so that any cubic containing $\Gamma^{\prime}$ contains $\Gamma$ (indeed $m \geq n+2$ ). We would like to show that this implies

$$
\left\{p_{1}(\alpha), \ldots, p_{m+1}(\alpha), p_{m+2}, \ldots, p_{d}\right\}
$$


fails to be cubically independent, i.e., that there is some arrow $p_{m+1}\left(l_{m+1}\right) \subset$ $p_{i}(\alpha)$ for which any cubic vanishing on

$$
\left\{p_{1}(\alpha), \ldots, p_{m}(\alpha), p_{m+1}, \ldots, p_{d}\right\}
$$

must vanish on $p_{m+1}\left(l_{m+1}\right)$. By Lemma 18 this implies that $m+d \geq 3 n+1$, with equality if and only if all the points are on a rational normal curve.

Since any cubic vanishing on $\Gamma^{\prime}$ also vanishes on all of $\Gamma$, for each $i$ we may find an arrow $p_{i}\left(l_{i}\right) \subset p(\alpha)$ so that

$$
\left\{p_{1}(\alpha), \ldots, p_{m}(\alpha), p_{m+1}\left(V_{d+1}\right), \ldots, p_{d}\left(V_{d}\right)\right\}
$$

is 3-independent provided that $V_{i} \nsupseteq l_{i}$. Fix some such $V_{m+1}, \ldots, V_{d-2}$, and let

$$
\Sigma=\left\{p_{1}(\alpha), \ldots, p_{m}(\alpha), p_{m+1}\left(V_{m+1}\right), \ldots, p_{d-2}\left(V_{d-2}\right)\right\}
$$

We have that $\Sigma \cup\left\{p_{d-1}\left(V_{d-1}\right), p_{d}\left(l_{d}\right)\right\}$ is cubically dependent for all $V_{d-1} \nsupseteq$ $l_{d-1}$ and hence it is 3-dependent for every $V_{d-1}$. So either $\Sigma \cup\left\{p_{d-1}, p_{d}\left(l_{d}\right)\right\}$ is 3-dependent, or any cubic containing $\Sigma \cup\left\{p_{d-1}, p_{d}\left(l_{d}\right)\right\}$ contains $p_{d-1}\left(l_{d-1}\right)$. But since any cubic containing $\Sigma \cup\left\{p_{d-1}, p_{d}(V)\right\}$ for $V \not \supset l_{d}$ also must contain $p_{d-1}\left(l_{d-1}\right)$, the latter implies that $\Sigma \cup\left\{p_{d-1}\left(l_{d-1}\right), p_{d}\right\}$ is 3-dependent. That is, $\Sigma \cup\left\{p_{d-1}, p_{d}\left(l_{d}\right)\right\}$ or $\Sigma \cup\left\{p_{d-1}\left(l_{d-1}\right), p_{d}\right\}$ is 3-dependent, so by uniform position both of these subschemes are 3-dependent.

Applying a similar argument to each

$$
\left\{p_{1}(\alpha), \ldots, p_{m}(\alpha), p_{m+1}\left(V_{m+1}\right), \ldots, p_{i}\left(V_{i}\right), p_{i+1}, \ldots, p_{d}\right\}
$$

for $i \geq m$ we get that every cubic vanishing on

$$
\left\{p_{1}(\alpha), \ldots, p_{m}(\alpha), p_{m+1}\left(l_{m+1}\right), \ldots, p_{d}\right\}
$$

vanishes on $p_{m+1}\left(L_{m+1}\right)$, as anticipated.

Induction step. Assume that a subscheme of $\Gamma$ consisting of $n+2$ dots of degree $\alpha$ and $(r-2) n-3$ dots of degree $(\alpha-1)$ imposes independent conditions in degree $r-1$. If $d \geq(r-1) n-1$ then a subscheme consisting of two $\alpha$-dots, $n$ reduced points, and $(r-1) n-3(\alpha-1)$-dots also imposes independent conditions in degree $r-1$. (To see this, by Corollary 8 it is enough to know that two $\alpha$-dots together with $(r-1) n-3$ reduced points is $(r-1)$-independent, which follows from Corollary 18 and induction.) Hence, by promotion, $n+2$ $\alpha$-dots and $(r-1) n-3(\alpha-1)$-dots imposes independent conditions in degree $r$. Thus, if $d \geq(r-1) n-1$ we have

$$
h_{\Gamma}(r) \geq r n+1+(\alpha-2)((r-1) n-1) .
$$

If $(r-2) n-1 \leq d<(r-1) n-1$, let $s=d-(r-2) n+1$. Then at least $n+2-s \quad \alpha$-dots, $s$ reduced points, and $d-n-2(\alpha-1)$-dots is $(r-1)$ independent, Now in degree $r$ let us promote the $s$ reduced points and $n-s$ of the $(\alpha-1)$-dots to get an $r$-independent subscheme consisting of $2 n+2-s$ 
$\alpha$-dots and $d-(2 n+2-s)(\alpha-1)$-dots for a total of

$$
\begin{aligned}
2 n-s+2+(\alpha-1) d & =2 n-d+(r-2) n+1+(\alpha-1) d \\
& =r n+1+(\alpha-2) d
\end{aligned}
$$

conditions. For each subsequent $r$, we can promote $n$ of the $(\alpha-1)$-dots, hence for every $r$ with $d \leq(r-1) n-1$ we get

$$
h_{\Gamma}(r) \geq \min (r n+1+(\alpha-2) d, \alpha d) \text {. }
$$

So, we get the

Theorem 20. Let $\Gamma$ be a collection of $d \geq n+1 \alpha$-dots in uniform position in $\mathbb{P}^{n}, \alpha \geq 2$. Then the Hilbert function $h_{\Gamma}$ of $\Gamma$ satisfies $h_{\Gamma} \geq h_{\alpha}$ where

$$
h_{\alpha}(r)= \begin{cases}n+1, & \text { if } r=1, \\
2 n+1+(\alpha-2)(n-1)-\left(\begin{array}{c}
\alpha-2 \\
2
\end{array}\right), & \text { if } r=2, \\
\min (r n+1,2 d)+(\alpha-2) \min ((r-1) n-1, d), & \text { if } r \geq 3\end{cases}
$$

In particular, as we'll see in section 4 , for $(r-1) n-1 \leq d$ the Hilbert function of $\Gamma$ is bounded below by the minimal Hilbert function of an $(\alpha-1)$ rope in $\mathbb{P}^{n}$.

\section{Hilbert fUnCtions OF ROPES}

Ropes over rational normal curves. In the previous section we obtained a lower bound for the Hilbert function of a collection $\Gamma$ of dots in uniform position and showed that if that bound is attained then the reduced points of $\Gamma$ lie on a rational normal curve. Let us now see that equality can actually occur, by looking at collections of dots whose reduced points lie on a rational normal curve.

Let $C$ be a rational normal curve in $\mathbb{P}^{n}$, and let $\mathscr{N}$ be the normal bundle of $C$ in $\mathbb{P}^{n}$. For any $p \in C$ we may define a line bundle $\mathscr{L}_{p}$ so that a section of $\mathscr{L}_{p}$ over a point $q \in C, q \neq p$ is the line between $p$ and $q$ in $\mathbb{P}^{n}$. Then, if $p_{1}, \ldots, p_{n-1} \in C$ are distinct points then $\mathscr{N}=\mathscr{L}_{p_{1}} \oplus \ldots \oplus \mathscr{L}_{p_{n-1}}$. We have $\operatorname{deg} \mathscr{L}_{p}=n+2$ and $\mathscr{L}_{p}$ has the maximal degree $n+2$ among all line subbundles of $\mathscr{N}$.

Consider the ribbon $X$ associated to $\mathscr{L}_{p}$. Then $\operatorname{deg} X=2 n$ and $g(X)=$ $n+1$ so the Hilbert polynomial of $X$ is $p_{X}(k)=(2 k-1) n$. On the other hand, by taking $n+1$ general 3-dots on $X$ we see that $h_{X}(2)=3 n$, and hence $h_{X}(r)=p_{X}(r)$ for $r \geq 2$.

We refer to the ribbon $X$ as a "canonical" ribbon over $C$. Indeed, $X$ is a canonical curve, except for the fact that it is nonreduced. That is, $X$ is a one-dimensional nondegenerate subscheme of $\mathbb{P}^{n}$ of degree $2 n$ and genus $n+1$.

Now, take the "canonical" ribbons $X_{1}, \ldots, X_{n-1}$ corresponding to direct summands $\mathscr{L}_{p_{1}}, \ldots, \mathscr{L}_{p_{n-1}}$. Let $W_{1}, \ldots, W_{n-1}$ be the spaces of quadrics vanishing on $X_{1}, \ldots, X_{n}$ respectively. If $W$ is the vector space of quadrics 
vanishing on $C$ then we have

$$
\begin{aligned}
\operatorname{codim}\left(W_{i}\right) & =n-1, \\
\operatorname{codim}\left(W_{i} \cap W_{j}\right) & \geq 2 n-3, \\
\operatorname{codim}\left(W_{i_{1}} \cap \ldots \cap W_{i_{j}}\right) & \geq \sum_{k=n-j}^{n-1} k, \\
& \vdots \\
\operatorname{codim}\left(W_{i_{1}} \cap \ldots \cap W_{i_{n-3}}\right) & \geq \operatorname{dim} W-3, \\
\operatorname{codim}\left(W_{i_{1}} \cap \ldots \cap W_{i_{n-2}}\right) & =\operatorname{dim} W-1,
\end{aligned}
$$

and

$$
\operatorname{codim}\left(W_{1} \cap \ldots \cap W_{n-1}\right)=\operatorname{dim} W .
$$

(That we really have equality in the last two inequalities is a consequence of the $\mathbb{P}^{3}$-lemma and its corollary in section 3.) lt follows that each of these inequalities is an equality. So an $\alpha$-rope $X_{\alpha}$ corresponding to $\alpha-1$ direct summands $\mathscr{L}_{p_{i}}$ has Hilbert function

$$
h_{X_{\alpha}}(2)=p_{X_{\alpha}}(2)-\left(\begin{array}{c}
\alpha-1 \\
2
\end{array}\right)
$$

which is exactly the lower bound that we found for the Hilbert function of $(\alpha+1)$-dots. A similar calculation shows that

$$
h_{X_{\alpha}}(r)=p_{X_{\alpha}}(r)
$$

for $r \geq 3$.

Thus, a collection of $\alpha$-dots lying on $X_{\alpha-1}$ will have the Hilbert function given in Theorem 20. Conversely, suppose that a collection $\Gamma$ of $\alpha$-dots has Hilbert function that agrees with the minimal Hilbert function for some $r$. If $h_{\Gamma}(r)=h_{X_{\alpha-1}}(r)$ for some $r$ with $(r-1) n-1 \leq d$ then by our estimate of the growth of $h_{\Gamma}$ we have that the same holds for $r=3$. We have seen that a collection of dots in uniform position having this Hilbert function must have reduced points that lie on a rational normal curve $C$. We may then find an $(\alpha-1)$-rope $X$ containing some $\min (2 n, d) \alpha$-dots of $\Gamma$.

Suppose $C \subset \mathbb{P}^{n}$ is a rational normal curve and that $p_{1}, \ldots, p_{2 n} \in C$ are points on $C$. Let $\Gamma=\left\{p_{1}\left(V_{1}\right), \ldots, p_{2 n}\left(V_{2 n}\right)\right\}$ be a collection of 3 -dots at the points $p_{i}$.

Lemma 21. There is a ribbon $X$ supported on $C$ containing $\Gamma$, corresponding to a line bundle $\mathscr{L} \cong \mathscr{O}_{\mathbb{P}_{1}}(m)$, where $2 \leq m \leq n+2$

Proof. To find such a ribbon $X$ of genus $m-1$ over $C$ is to find a line bundle $\mathscr{L} \cong \mathscr{O}_{\mathbb{P}^{1}}(m) \subset \mathscr{N}$ for which $\left.\mathscr{L}\right|_{p_{i}}=V_{i}$

Since $\mathscr{N} \cong \mathscr{O}_{\mathbb{P l}}(n+2) \otimes V$, where $\operatorname{dim} V=n-1$, the data of such a line bundle is equivalent to that of its Gauss map $\phi: \mathbb{P}^{1} \rightarrow \mathbb{P} V=\mathbb{P}^{n-2}$, where $\phi\left(p_{i}\right)=\left[V_{i}\right]$ for $i=1, \ldots, 2 n$, which is then of degree $d=\operatorname{deg} \phi=n+2-m$.

We seek a $(n-1)$-tuple $\phi=\left(\phi_{1}, \ldots, \phi_{n-1}\right)$ of homogeneous polynomials on $\mathbb{P}^{1}$, each of degree $d$ (or less), having prescribed values at $2 n$ points. 
Taking each pair of coordinates separately, then we look for some $(n-2)$ maps from $\mathbb{P}^{1}$ to $\mathbb{P}^{1}$ with prescribed values at $2 n$ points. Each time this is possible if the degree $d$ has $2(d+1)>2 n$ or $d \geq n$. Hence we can find an appropriate Gauss map of degree $d \leq n$, i.e., $m=n+2-d \geq 2$.

We have

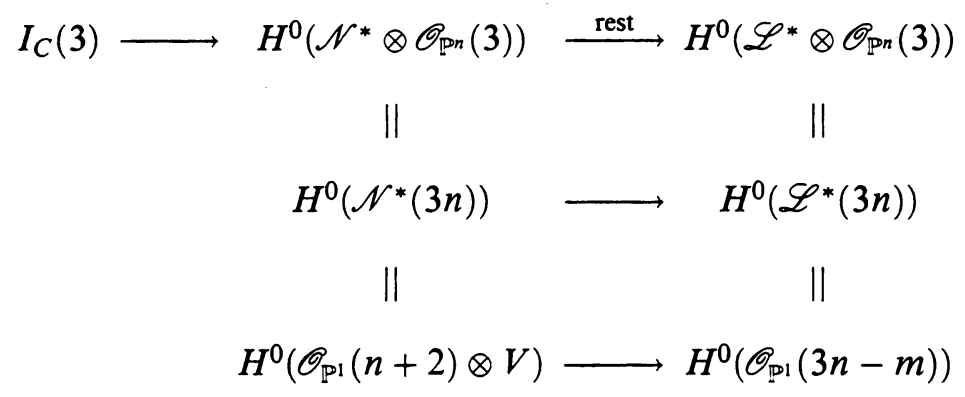

We have seen that the map $I_{C}(3) \stackrel{\text { rest }}{\longrightarrow} H^{0}\left(\mathscr{N}^{*} \otimes \mathscr{O}_{\mathbb{P} n}(3)\right)$ is surjective (since the Hilbert function of the $n$-rope over $C$ agrees with its Hilbert polynomial in degree 3), whereas the map $H^{0}\left(\mathscr{N}^{*}(3 n)\right) \rightarrow H^{0}\left(\mathscr{L}^{*}(3 n)\right)$ (multiplication) is surjective for $m \geq 2$.

Now since the map $I_{C}(3) \rightarrow H^{0}\left(\mathscr{O}_{\mathbb{P 1}}(3 n-m)\right)$ is surjective, then the points $p_{1}, \ldots, p_{2 n}$ fail to impose independent conditions on the linear series $\left|\mathscr{O}_{\mathbb{P} 1}(3 n-m)\right|$ if and only if $3 n-m \leq 2 n-2$, i.e., $m \geq n+2$.

We may put any $2 n$ dots of $\Gamma$ on an $(\alpha-1)$-rope $X$ corresponding to some subbundle $\mathscr{E} \doteq \mathscr{O}_{\mathbb{P} 1}\left(m_{1}\right) \oplus \cdots \oplus \mathscr{O}_{\mathbb{P 1}}\left(m_{\alpha-1}\right)$ of the normal bundle of $C$ in $\mathbb{P}^{n}$. Then

$$
2 \alpha n-h_{\Sigma}(3)=\#\left\{i: m_{i}=n+2\right\}
$$

and in particular $h_{\Gamma}=3 n+1+(\alpha-2)(2 n-1)$ if and only if $\Gamma$ is contained in a ribbon corresponding to a direct sum of $\mathscr{O}_{\mathrm{Pl}}(n+2)$ 's.

Likewise, if any one of these direct summands fails to be $\mathscr{O}_{\mathbb{P l}}(n+2)$, then we have

$$
h_{\Gamma}(2)=h_{X}(2) \geq 2 n+1+(\alpha-2)(n-1)-\left(\begin{array}{c}
\alpha-2 \\
2
\end{array}\right)+1 .
$$

In summary, we get the

Theorem 22. Let $\Gamma$ be a collection of $d \alpha$-dots in uniform position in $\mathbb{P}^{n}$, $\alpha \geq 2$. Then the Hilbert function $h_{\Gamma}$ of $\Gamma$ satisfies

$$
h_{\Gamma} \geq \min \left(h_{X_{\alpha-1}}(r), r n+1+(\alpha-2) d, \alpha d\right)
$$

where $X_{\alpha-1}$ is the $(\alpha-1)$-rope described above. We have equality for some $r$ with $(r-1) n-1 \leq d$ if and only if $\Gamma$ is contained in an $X_{\alpha-1}$. Equality occurs for some $r$ with $r n+1 \leq 2 d$ if and only if $\Gamma_{\text {red }}$ is contained in a rational normal curve, and the tangent directions to the curve at these points are all contained in $\Gamma$. 
The Hilbert function of a rope. Let $X \subset \mathbb{P}^{n}$ be an $\alpha$-rope over a curve of degree $d$. Then for each $r$ we have

$$
h_{X}(r) \geq h_{X}(r-1)+h_{X \cap H}(r),
$$

so

$$
\begin{aligned}
h_{X}(r) & \geq h_{X}(2)+\sum_{k=3}^{r} h_{X \cap H}(k), \\
\geq & \sum_{k=1}^{r}(\min (k(n-1)+1,2 d)+(\alpha-2) \min ((k-1)(n-1)-1, d)) \\
& \cdot-\left(\begin{array}{c}
\alpha-2 \\
2
\end{array}\right)+\alpha-1 .
\end{aligned}
$$

Thus, we get

Proposition 23. Let $X$ be a $\alpha$-rope over a curve $C$ of degree $d$ in $\mathbb{P}^{n}$. Let $m=\left[\frac{d+1}{n-1}\right]$ and $M=\left[\frac{2 d-1}{n-1}\right]$. Then the Hilbert function $h_{X}$ of $X$ satisfies the following lower bound. For $2 \leq k \leq m+1$ (i.e., $(k-1)(n-1) \leq d)$,

$$
h_{X}(k) \geq(\alpha-2) \sum_{r=0}^{k-1}(r(n-1)-1)+\sum_{r=1}^{k}(r(n-1)+1)-\left(\begin{array}{c}
\alpha-2 \\
2
\end{array}\right)+\alpha-1 .
$$

For $m+2 \leq k \leq M$ (i.e., $(k-1)(n-1)>d$ and $k(n-1)+1 \leq 2 d)$,

$$
\begin{aligned}
h_{X}(k) \geq & (\alpha-2) \sum_{r=0}^{m}(r(n-1)-1)+\sum_{r=1}^{k}(r(n-1)+1)+(k-m-1)(\alpha-2) d \\
& -\left(\begin{array}{c}
\alpha-2 \\
2
\end{array}\right)+\alpha-1 .
\end{aligned}
$$

For $k \geq M+1$,

$$
\begin{aligned}
h_{X}(k) \geq & (\alpha-2) \sum_{r=0}^{m}(r(n-1)-1)+\sum_{r=1}^{M}(r(n-1)+1) \\
& +(M-m-1)(\alpha-2) d+(k-M) \alpha d-\left(\begin{array}{c}
\alpha-2 \\
2
\end{array}\right)+\alpha-1 .
\end{aligned}
$$

Corollary 24. Let $G$ be the genus of an $\alpha$-rope $X \subset \mathbb{P}^{n}$ on a curve of degree $d$. Let $m=\left[\frac{d+1}{n-1}\right]$ and $M=\left[\frac{2 d-1}{n-1}\right]$, and write $d=m(n-1)-1+\delta$, $2 d=M(n-1)+1+\nu$, where $0 \leq \delta, \nu \leq n-1$. Then

$$
\begin{aligned}
G \leq & (n-1)\left((\alpha-2)\left(\begin{array}{c}
m+1 \\
2
\end{array}\right)+\left(\begin{array}{c}
M \\
2
\end{array}\right)\right)+(\alpha-2)(m+1) \delta+M \nu \\
& +2-\alpha+\left(\begin{array}{c}
\alpha-2 \\
2
\end{array}\right)
\end{aligned}
$$

with equality only if the general hyperplane section of $X$ lies on an $(\alpha-1)$-rope, of maximal genus, over a rational normal curve. 
Remark. The maximal $G$ depends on $d, n, \alpha$ as, roughly $G \leq(\alpha+2)$. $\left(\frac{d^{2}}{2 n-2}+d-1\right)$, and in case $G$ approaches this bound then $G$ is approximately $(\alpha+2)(g+d-1)$ where $g$ is the (maximal) genus of the underlying reduced curve of $X$.

Proof. If $m=\left[\frac{d+1}{n-1}\right], M=\left[\frac{2 d-1}{n-1}\right]$ and $d=m(n-1)-1+\delta, 2 d=$ $M(n-1)+1+\nu$ we have

$$
\begin{aligned}
G= & M \alpha d+1-h_{X}(M) \\
\leq & M \alpha d+1-(\alpha-2) \sum_{r=1}^{m}(r(n-1)-1)-\sum_{r=0}^{M}(r(n-1)+1) \\
& -(M-m-1)(\alpha-2) d+\left(\begin{array}{c}
\alpha-2 \\
2
\end{array}\right)-\alpha+1 . \quad
\end{aligned}
$$

Corollary 25. Let $C$ be a smooth irreducible nondegenerate curve of degree $d$ and genus $g$ in $\mathbb{P}^{n}$ and let $\mathscr{N}=\mathscr{N}_{C, \mathbb{P} n}$ be its normal bundle. If $S \subset \mathscr{N}$ is a subbundle of rank $\alpha$ then

$$
\begin{aligned}
\operatorname{deg} S \leq & (n-1)\left(\left(\begin{array}{c}
M \\
2
\end{array}\right)+(\alpha-1)\left(\begin{array}{c}
m+1 \\
2
\end{array}\right)\right)+(\alpha-1)(m+1) \delta+M \nu \\
& -\alpha+(\alpha+1)(1-g)+\left(\begin{array}{c}
\alpha-1 \\
2
\end{array}\right)
\end{aligned}
$$

with equality only if the genus $g$ of $C$ is maximal with respect to $d$ and $n$.

Remarks. The bound obtained in Theorem 23 is not sharp. Even in the case where $X$ is an $\alpha$-rope over a rational normal curve, we have seen that this bound is off by $\left(\begin{array}{c}\alpha-1 \\ 2\end{array}\right)$. We might expect, for example, that a $n$-rope $X$ is in $\mathbb{P}^{n}$ over a curve of degree $\geq 2 n-3$ will have the smallest Hilbert function if and only if the curve lies on a rational normal surface scroll $S$, in which case $h_{X}(3)=3 n^{2}-6 n+11$, which is the Hilbert function of the double scroll.

If so, an $\alpha$-rope $X$ has minimal possible Hilbert function if and only if $C=X_{\text {red }}$ is a Castelnuovo curve and its general hyperplane section lies on a $(\alpha-1)$-rope of maximal genus over a rational normal curve. But is there, for each $\alpha$, such an $\alpha$-rope? For $\alpha=n$, the answer is yes: take the full rope over a Castelnuovo curve, corresponding to the entire normal bundle of the curve in $\mathbb{P}^{n}$. For $\alpha=2$, take the normal bundle of the curve with repect to the surface scroll $S$ it lies on. For $\alpha=3$, there is also an appropriate $\alpha$-rope over $C$. Namely, the normal bundle of the scroll $S$ has a (unique) direct summand that restricts to $\mathscr{O}_{\mathrm{P} 1}(n+2)$ on the rational normal curve given as its general hyperplane section.

We should also note that while Theorem 23 gives a uniform bound on the Hilbert function of an $\alpha$-rope $X$ over any curve $C$ of degree $d$ in $\mathbb{P}^{n}$ (and, respectively, Corollary 24 gives a bound on its genus), regardless of the genus $g$ of $C$, this bound will only be attained if the genus of $C$ is itself maximal. It should be possible to get a still more general bound on the Hilbert function of $X$ in terms of $d, n$, and $g$ so that we can obtain a reasonable estimate in case the genus of $C$ is known. 


\section{REFERENCES}

[AH] J. Alexander and A. Hirschowitz, La méthode d'Horace éclatée: application à l'interpolation en degré quatre, Invent. Math. 107 (1992), 585-602.

[ACGH] E. Arbarello, M. Cornalba, P.A. Griffiths, and J. Harris, Geometry of algebraic curves, Vol. I, Springer-Verlag, New York, 1985.

[BE] D. Bayer and D. Eisenbud, Ribbons and their canonical embeddings, Trans. Amer. Math. Soc. 347 (1995), 757-765.

[C1] K. Chandler, Hilbert functions of dots in uniform position, Ph.D. Thesis, Harvard Univ., 1992.

[C2] Hilbert functions of dots in linear general position, Proc. Conference on ZeroDimensional Schemes, Ravello, Italy, June, 1992.

[EH1] D. Eisenbud and J. Harris, Finite projective schemes in linearly general position, J. Algebraic Geom. 1 (1992), 1-40.

[EH2] D. Eisenbud and J. Harris, Schemes: The language of algebraic geometry, Wadsworth and Brooks, Belmont, Calif., 1992.

[H1] J. Harris (with D. Eisenbud), Curves in projective space, Les Presses de l'Université de Montréal, Montréal, Québec, Canada, 1982.

[H2] J. Harris, Algebraic geometry: A first course, Graduate Texts in Math., 33, SpringerVerlag, 1992.

Department of Mathematics, University of Chicago, Chicago, Illinois 60637

E-mail address: kac@math. uchicago.edu 